\title{
Dynamical Transition of Protein-Hydration Water
}

\author{
W. Doster, ${ }^{*}$ S. Busch, and A. M. Gaspar \\ Physik Department E 13 and ZWE FRM II, Technische Universität München, 85747 Garching, Germany
}

M.-S. Appavou and J. Wuttke

Forschungszentrum Jülich, JCNS at FRM II, Lichtenbergstrasse 1, 85747 Garching, Germany

\author{
H. Scheer \\ Botanisches Institut II, Ludwig-Maximilians-Universität München, Menzinger Strasse 67, 80638 München, Germany \\ (Received 22 July 2009; published 2 March 2010)
}

\begin{abstract}
Thin layers of water on biomolecular and other nanostructured surfaces can be supercooled to temperatures not accessible with bulk water. Chen et al. [Proc. Natl. Acad. Sci. U.S.A. 103, 9012 (2006)] suggested that anomalies near $220 \mathrm{~K}$ observed by quasielastic neutron scattering can be explained by a hidden critical point of bulk water. Based on more sensitive measurements of water on perdeuterated phycocyanin, using the new neutron backscattering spectrometer SPHERES, and an improved data analysis, we present results that show no sign of such a fragile-to-strong transition. The inflection of the elastic intensity at $220 \mathrm{~K}$ has a dynamic origin that is compatible with a calorimetric glass transition at $170 \mathrm{~K}$. The temperature dependence of the relaxation times is highly sensitive to data evaluation; it can be brought into perfect agreement with the results of other techniques, without any anomaly.
\end{abstract}

DOI: 10.1103/PhysRevLett.104.098101

PACS numbers: 64.70.pm, 87.15.H-

In contrast to bulk water, protein-hydration water can be supercooled down to a glass transition at $T_{g} \simeq 170 \mathrm{~K}$. Near $T_{g}$ translational degrees of freedom arrest, which induces discontinuities in the specific heat and the thermal expansion coefficient of the hydration water [1-4]. Because of the dynamic nature of the glass transition, freezing of microscopic degrees of freedom can already be observed far above $T_{g}$.

The protein dynamic transition is an abrupt onset of atomic displacements on the microscopic length and time scale probed by quasielastic neutron scattering (QENS). First observed 20 years ago in hydrated myoglobin and lysozyme at $T_{\Delta} \simeq 240 \mathrm{~K}$ [5], it is now known to be a generic property of hydrated proteins, while it is absent in dehydrated systems. It is therefore related to the dynamics of the hydration shell.

In QENS, mean squared dispacements $\left\langle\delta x^{2}\right\rangle$ are deduced from the elastic scattering intensity $S(q, 0)$. Because of the finite spectrometer resolution (FWHM $2 \Delta \omega)$, one actually measures $S(q,|\omega| \lesssim \Delta \omega)$. Full spectral measurements show that the anomalous decrease of the central peak is compensated by increasing inelastic wings $[5,6]$. These effects have been interpreted as precursors of the glass transition. Since QENS probes structural relaxation at $\Delta \omega^{-1} \simeq 100 \mathrm{ps}$, while the calorimetric $T_{g}$ refers to a time scale of $100 \mathrm{~s}$, it is natural that $T_{\Delta}$ is located far above $T_{g}$ : the protein dynamic transition is the microscopic manifestation of the glass transition in the hydration shell. The time-scale dependence of $T_{\Delta}$ also explains its variation with viscosity [7-9].

Recently, these views have been challenged by the suggestion that there might be a time-scale independent tran- sition. Support came mainly from QENS experiments on the backscattering spectrometer HFBS at NIST. In hydrated lysozyme, DNA and RNA, a kink was found not only in $S(q,|\omega| \lesssim \Delta \omega)$, but also in the $\alpha$ relaxation time $\langle\tau\rangle$ deduced from full spectra $S(q, \omega)$ [10-13]. This change of $\langle\tau\rangle(T)$ from high- $T$ super-Arrhenius to low- $T$ Arrhenius behavior at $T_{L} \simeq 220 \mathrm{~K}$ has been interpreted as a fragileto-strong-transition (FST) from the high density (HDL) to the less fluid low density phase (LDL) of supercooled water [14]. In this view, a qualitative change in the dynamics occurs when the so-called Widom line is crossed, which extrapolates the phase boundary beyond the conjectured critical point $[15,16]$.

Similar behavior was found for confined water in various nonbiological environments [[17,18] and references therein]; $T_{L}$ was mostly located between 215 and $228 \mathrm{~K}$. There were, however, two remarkable exceptions: making a substrate hydrophobic lowered $T_{L}$ by $35 \mathrm{~K}$ [19]; using another neutron spectrometer with wider dynamic range lowered $T_{L}$ by about 10 to $20 \mathrm{~K}$ [20]. Critics objected that relaxation times measured by other spectroscopic techniques show no anomaly at $T_{L}$ [21-23].

In this Letter we will argue that the changing temperature dependence of $\log (\langle\tau\rangle)$ is likely to be an artifact of the data analysis, and that the kink in the elastic intensity also admits a conventional, dynamic interpretation. We will refer to the hydration shell of different proteins. If the anomalies observed around $T_{L}$ were due to an intrinsic property of water, then the choice of the protein should not matter.

To improve the empirical base, we measured the motion of protein-hydration water with enhanced sensitivity, using 
a new neutron spectrometer and a fully deuterated protein. We chose deuterated C-phycocyanin (D-CPC), a light harvesting, blue copper protein. Deuteration drastically reduces scattering from the protein so that we become more sensitive to weak quasielastic scattering from the hydration water. CPC has been investigated by neutron scattering before [24-28]. The sample was purified from a preparation of Crespi $[28,29]$ with nearly $99 \%$ deuteration. The absorbance ratio $A_{620 \mathrm{~nm}} / A_{280 \mathrm{~nm}} \simeq 7.3$ is above the analytic-grade value of 5 [30]. The integrity of the secondary structure was ascertained by CD spectroscopy. The protein was dialyzed against distilled water, freeze dried, and rehydrated to $h=0.3 \mathrm{~g} / \mathrm{g}$ [27], the same degree of hydatrion as in Ref. [10].

QENS was performed on $500 \mathrm{mg}$ of hydrated D-CPC with the new backscattering spectrometer SPHERES at FRM II [31]. It has a resolution (FWHM) of $0.62 \mu \mathrm{eV}$ (HFBS $0.85 \mu \mathrm{eV}$ ), high flux, and an outstanding signal-tonoise ratio of 1000:1. Scattering wave numbers $q$ range from 0.2 to $1.8 \AA^{-1}$. We chose about the same energy range $\pm 10 \mu \mathrm{eV}$ as customary at HFBS. Spectra at temperatures $T$ between 100 and $300 \mathrm{~K}$ were collected for 6 to $12 \mathrm{~h}$. An empty cell measurement was subtracted and spectra were normalized to a vanadium standard. The analysis was performed with the program FRIDA [32].

Figure 1 displays selected spectra in the relevant temperature range. The logarithmic intensity scale emphasizes both the broad, asymmetric wing of the instrumental resolution function, unavoidable in a crystal spectrometer, and the quasielastic scattering. At least two distinct components of the scattering function $S(q, \omega)$ can be observed: (i) An elastic component that has the shape of the instrumental resolution function $R_{q}(\omega)$. It is mainly due to the

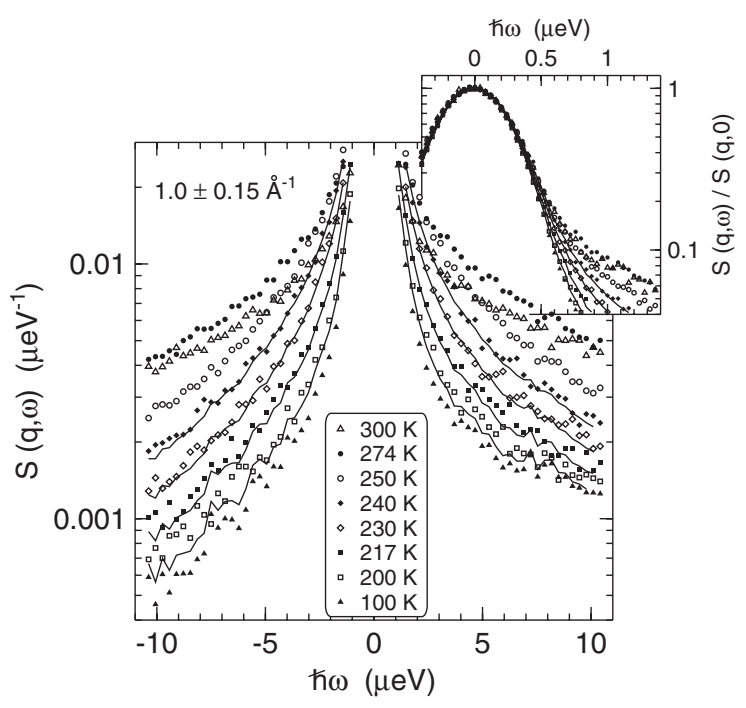

FIG. 1. Neutron scattering spectra of $\mathrm{H}_{2} \mathrm{O}$ on D-CPC at $q=$ $1.0 \pm 0.15 \AA^{-1}$. Note the truncated logarithmic scale. Solid lines are fits with (1), convoluted with the instrumental resolution (sample data at $100 \mathrm{~K}$ ).- - Inset: central peak, normalized to the value at $\omega=0$. coherent structure factor of the protein (as evidenced by strong small-angle scattering for $q \ll 1 \AA^{-1}$ [27]), as well as to a protein incoherent term mainly due to the exchanged protons $(25 \%)$. Also immobilized water near charged groups contributes to this component. (ii) A quasielastic component that appears above about $170 \mathrm{~K}$. It is mainly due to motion of adsorbed $\mathrm{H}_{2} \mathrm{O}$ molecules.

To start with a model-free data characterization, we examined the $T$ dependence of the integrated inelastic intensity for different $\omega$ intervals, preferentially on the $\omega<0$ side where the resolution wing is weaker. Between 180 and $230 \mathrm{~K}$, the inelastic intensity begins to rise strongly. There is no sharp kink; the temperature of maximum inflection depends on the $\omega$ range and on $q$ [Fig. 2(a)]. This is a first indication for a dynamic phenomenon rather than a hidden phase transition: the $q$ and $T$ dependence is compatible with a smooth onset of translational diffusion. The elastic intensity in compensation shows a steplike decrease at $220 \mathrm{~K}$ in parallel with data obtained with lysozyme. This suggests a common transi-

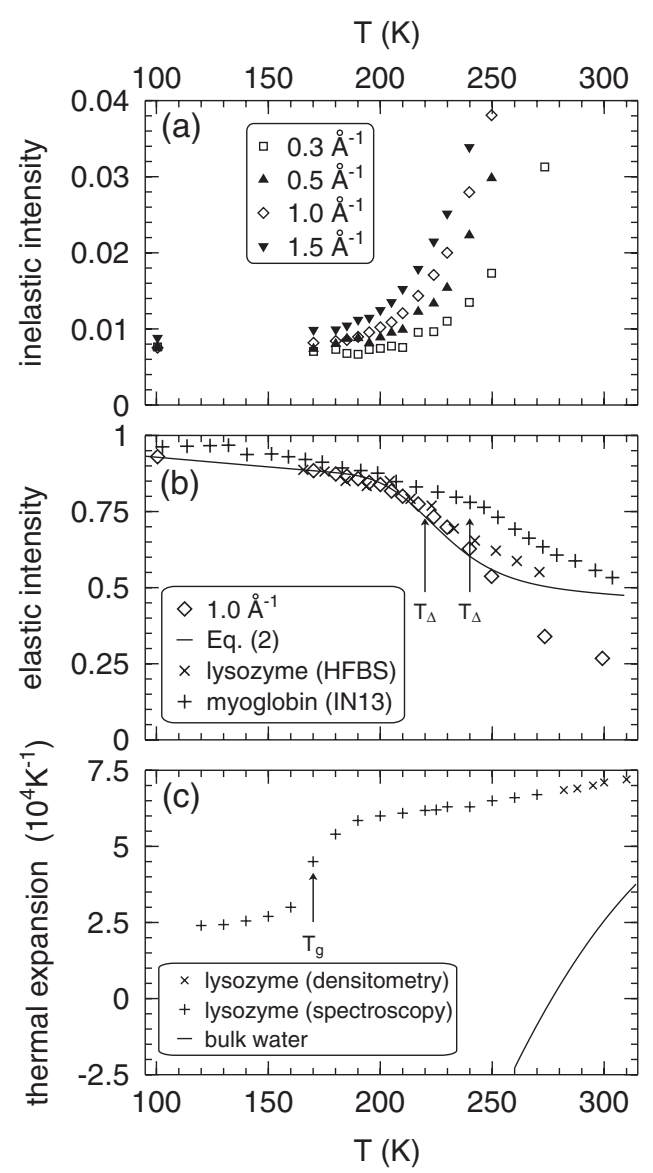

FIG. 2. (a) Scattering intensity integrated over $\hbar \omega<-3 \mu \mathrm{eV}$. (b) Elastic scattering intensity $S(q, 0)$ and result of (2), compared to literature data $[5,10]$. (c) Thermal expansion coefficient $\alpha_{P}$ of water in hydrated lysozyme powder $(+$, spectroscopic measurement [3]), of hydration water in lysozyme solution $(\times$, densitometry, [36]), of bulk water (line). 
tion temperature of both hydrated proteins [Fig. 2(b)]. In the following, we concentrate on $q=1.0 \pm 0.15 \AA^{-1}$.

To analyze the spectra, we use the same model as Chen et al. [10], consisting of an elastic line and a Kohlrausch-Williams-Watts function (KWW, Fourier transform of a stretched exponential [33]):

$$
S_{\mathrm{th}}(q, \omega)=f_{q}\left\{a_{1} \delta(\omega)+a_{2} \int_{-\infty}^{\infty} \frac{d t}{2 \pi} e^{i \omega t-(|t| / \tau)^{\beta}}\right\} .
$$

The Debye-Waller factor $f_{q}=\exp \left(-\left\langle\delta x^{2}\right\rangle q^{2}\right)$ accounts for phonon scattering outside our energy window. The mean squared displacement $\left\langle\delta x^{2}\right\rangle$ is assumed to be linear in $T$; from 100 and $170 \mathrm{~K}: \partial\left\langle\delta x^{2}\right\rangle / \partial T=7 \times 10^{-4} \AA^{2} / \mathrm{K}$. $\beta$ was fixed to 0.5 as in the analysis of Chen et al.

Equation (1) can fit the data sets for $T \leq 240 \mathrm{~K}$, hereby covering the temperature range of the proposed transition. At higher $T$, the data start to deviate from Eq. (1): additional quasielastic scattering, likely due to relaxational or fast anharmonic motion of the protein, is not included in this bimodal model.

In a first attempt to extract the relaxation times, we have used a standard fitting procedure for the data evaluation. Fitting the quasielastic wings for $T<240 \mathrm{~K}$, we are in the regime $\omega \tau \gg 1$, where the KWW function goes in first order with $(\omega \tau)^{-1-\beta}$. In such a power-law regime, the fit parameters amplitude and time-scale $\tau$ are degenerate. Therefore, the $240 \mathrm{~K}$ data were used to determine the relative contribution of the water $a_{2} /\left(a_{1}+a_{2}\right)$ to 0.42 which was then used as input for the other temperatures.

However, this approach neglects a severe limitation of the standard fitting procedure for narrow lines: When fitting the central peak, the convolution of $S_{\text {th }}$ with the experimental resolution $R_{q}(\omega)$ becomes nontrivial for $T \lesssim$ $220 \mathrm{~K}$ where the peak in the KWW function becomes so sharp (compared to the experimental energy binning) that the straightforward calculation $S_{\text {th }}(q, \omega) \otimes R_{q}(\omega)$ as a Riemann sum yields a wrong amplitude $a_{1}$. Because of the mentioned degeneracy of amplitude and time scale, this results in a distorted $\langle\tau\rangle$. We have therefore repeated the fits with an improved fitting procedure, neglecting the erroneous $a_{1}$ and fixing directly $a_{2}$ at 0.42 .

Figure 3 shows an Arrhenius plot of the mean relaxation time $\langle\tau\rangle=\int d t \exp (-t / \tau)^{\beta}=\beta^{-1} \Gamma\left(\beta^{-1}\right) \tau$ resulting from both fitting procedures. As can be seen, the temperature dependence of the relaxation times obtained from the standard fitting procedure resembles the results of Chen et al. This trend is however not observed any more in the results of the improved fitting procedure which agree well with the structural $(\alpha-)$ relaxation times of water on other proteins taken from literature (also shown). There is a good agreement with high- $T$ myoglobin data measured by QENS over a wider energy range than covered here [8] and for $T \lesssim 220 \mathrm{~K}$ with both, NMR [34] and dielectric spectroscopy [4] which show a smooth increase without inflection at $220 \mathrm{~K}$. The curvature of $\langle\tau\rangle$ in the Arrhenius

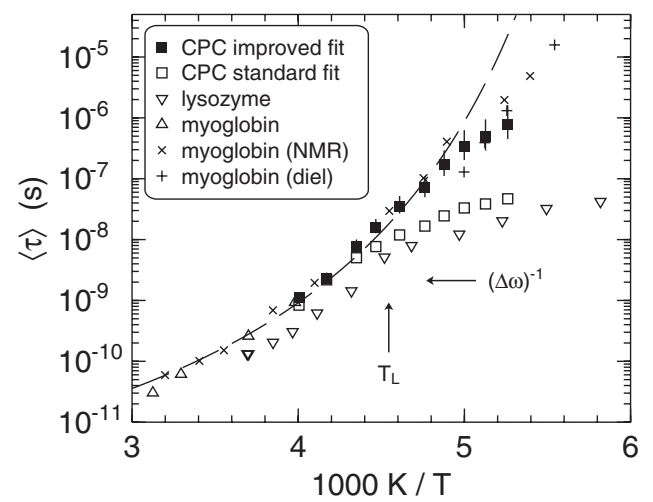

FIG. 3. Average relaxation times $\langle\tau\rangle$ versus reciprocal temperature for hydration water on different proteins: (ם): $c$-phycocyanin with improved fitting procedure and $(\square)$ : with a standard fitting procedure. Error bars were estimated by varying $\beta$ between 0.45 and 0.55 . $(\nabla)$ : lysozyme, QENS [10]. $(\triangle)$ : myoglobin, QENS with wide $\omega$ range [8]. $(\times)$ : myoglobin, NMR [34]. (+): myoglobin, dielectric loss [4], vertical arrow: conjectured $T_{L}$ of the FST, horizontal arrow: instrumental resolution $(\Delta \omega)^{-1}$. Dashed line: Vogel-Fulcher fit to the data above $200 \mathrm{~K}$ with fixed $T_{g}=170 \mathrm{~K}$, yielding $\langle\tau\rangle_{0}=6.5 \times 10^{-13} \mathrm{~s}$, $T_{H}=745 \mathrm{~K}$, and $T_{0}=147 \mathrm{~K}$.

plot indicates fragile behavior according to a VogelFulcher relation, $\langle\tau\rangle=\langle\tau\rangle_{0} \exp \left[T_{H} /\left(T-T_{0}\right)\right]$.

For low $T$, our $\langle\tau\rangle$ exceeds the resolution $\Delta \omega^{-1} \simeq 2 \mathrm{~ns}$ by some orders of magnitude. Still, for $|\hbar \omega| \gtrsim 2 \mu \mathrm{eV}$ there are clear quasielastic wings that allow to determine $\tau$ within the chosen model with fixed parameters $a_{2}$ and $\beta$. Therefore, this analysis can be used to probe the spectral behavior for the predicted FST discontinuity.

Whereas our first analysis, using the standard fitting procedure, reproduces the results from hydrated lysozyme [10] - the basis for the postulated FST at $220 \mathrm{~K}$-we can rule out any discontinuity at this temperature using the improved fitting procedure. We suspect therefore that also the various results backing the FST are affected by the problems of the standard fitting procedure: In the power-law regime $\omega \tau \gg 1$, fitted $\langle\tau\rangle$ are meaningful only if the amplitude $a_{2}$ has been fixed at a physically reasonable value. In the FST reports, the amplitude, if mentioned at all, is just called a scaling constant [17]. The fitted $\langle\tau\rangle(T)$ are therefore possibly distorted by an uncontrolled drift of $a_{2}(T)$. This could explain why the crossover temperature $T_{L}$ varies with pressure, while the crossover time appears constant [13].

Below approximately $200 \mathrm{~K}$, the relaxation times extracted from our improved technique deviate slightly from the Vogel-Fulcher relation. Being orders of magnitude above the instrumental resolution, a detailed discussion of this effect seems inadequate. If it were real, one could attribute it to the onset of the glass transition which is known to be about $30 \mathrm{~K}$ broad [4,35]. The observed process would then no longer be the isotropic $\alpha$ relaxation [34].

In contrast to the kink in $\langle\tau\rangle$, the kink in the observed elastic scattering intensity $S(q,|\omega| \lesssim \Delta \omega)$ is an undisput- 
able experimental fact. Figure 2(b) shows that observations at SPHERES (D-CPC) and HFBS (lysozyme [10]) almost coincide below $240 \mathrm{~K}$. The difference at higher $T$ can be explained by our use of deuterated CPC, which reduces the relative contribution of the protein.

The expected elastic scattering is

$$
\begin{aligned}
S_{\text {exp }}(q, 0) & =\int d \omega S_{\mathrm{th}}(q, \omega) R_{q}(-\omega) \\
& =f_{q}\left\{a_{1} R_{q}(0)+\left(1-a_{1}\right) \int_{0}^{\infty} \frac{d t}{\pi} e^{-(t / \tau)^{\beta}} \tilde{R}_{q}(t)\right\}
\end{aligned}
$$

with $\tilde{R}_{q}(t)=\int d \omega \cos (\omega t) R_{q}(\omega)$. Indeed, the low- $T$ data are perfectly reproduced by Eq. (2) using the values from the Vogel-Fulcher relation for $\langle\tau\rangle$. This confirms the long established explanation of the protein dynamic transition as a time-scale dependent glass transition: When $\tau^{-1}$ exceeds the instrumental resolution $\Delta \omega$, then the central part of the KWW spectrum starts to broaden so that $S(q,|\omega| \lesssim$ $\Delta \omega)$ falls below $f_{q}$.

This is further corroborated by elastic scattering $(q=$ $1.4 \AA^{-1}$ ) from hydrated myoglobin on the thermal neutron backscattering spectrometer IN13 [5]. Its resolution width $2 \hbar \Delta \omega=8 \mu \mathrm{eV}$ is an order of magnitude larger than that of HFBS or SPHERES, and the kink in $S(q,|\omega| \lesssim \Delta \omega)$ is observed accordingly at a higher temperature, namely, the often reported $T_{\Delta} \simeq 240 \mathrm{~K}$.

Previous QENS experiments were motivated by the hope that hydration layers would provide an opportunity to study water in a deeply supercooled state that is not accessible in the bulk. However, thin hydration layers seem to be qualitatively different from bulk water [21]. This view is also supported by literature data of the thermal expansion coefficient $\alpha_{P}$ of lysozyme hydration water [Fig. 2(c)]. In concentrated solution, $\alpha_{P}$ is nearly $T$ independent and much larger than the bulk value [36]. In hydrated powder, $\alpha_{P}$ is estimated from the $\mathrm{O}-\mathrm{H}$ stretching frequency of water, which varies with the average $\mathrm{H}$ bond length. A striking step is found near $170 \mathrm{~K}$, induced by the softening of the $\mathrm{O}-\mathrm{H}-\mathrm{O}$ hydrogen bond network [3]. No anomaly is observed at $T_{L} \simeq 220 \mathrm{~K}$.

All observations agree, however, with a glass transition of the hydration shell and a dynamic onset temperature $T_{\Delta}$, which varies with the probe frequency [Fig. 2(b)]. The different values of $\alpha_{P}$ for bulk and hydration water could indicate that the protein acts as a patch-breaker [37], suppressing critical fluctuations present in bulk water.

This work has been supported by Deutsche Forschungsgemeinschaft through SFB 533. We thank the late Henry Crespi for providing D-CPC.

*wdoster@ph.tum.de

[1] W. Doster, BBA Proteins and Proteomics 1804, 3 (2010).
[2] Y. Miyazaki, T. Matsuo, and H. Suga, J. Phys. Chem. B 104, 8044 (2000).

[3] F. Demmel, W. Doster, W. Petry, and A. Schulte, Eur. Biophys. J. 26, 327 (1997).

[4] H. Jansson and J. Swenson, BBA Proteins and Proteomics 1804, 20 (2010).

[5] W. Doster, S. Cusack, and W. Petry, Nature (London) 337, 754 (1989).

[6] W. Doster, S. Cusack, and W. Petry, Phys. Rev. Lett. 65, 1080 (1990).

[7] H. Lichtenegger et al., Biophys. J. 76, 414 (1999).

[8] W. Doster and M. Settles, BBA Proteins and Proteomics 1749, 173 (2005).

[9] W. Doster, Eur. Biophys. J. 37, 591 (2008).

[10] S.-H. Chen et al., Proc. Natl. Acad. Sci. U.S.A. 103, 9012 (2006).

[11] S.-H. Chen, L. Liu, and Y. Zhang, J. Chem. Phys. 125, 171103 (2006).

[12] X.-Q. Chu et al., Phys. Rev. E 77, 011908 (2008).

[13] X.-Q. Chu et al., J. Phys. Chem. B 113, 5001 (2009).

[14] P. Poole, F. Sciortino, U. Essmann, and H. E. Stanley, Nature (London) 360, 324 (1992).

[15] K. Ito, C. T. Moynihan, and C. A. Angell, Nature (London) 398, 492 (1999).

[16] L. Xu et al., Proc. Natl. Acad. Sci. U.S.A. 102, 16558 (2005).

[17] E. Mamontov et al., Chem. Phys. 352, 117 (2008).

[18] E. Mamontov et al., Phys. Rev. E 79, 051504 (2009).

[19] X.-Q. Chu et al., Phys. Rev. E 76, 021505 (2007).

[20] E. Mamontov et al., J. Phys. Chem. C 112, 12334 (2008).

[21] J. Swenson, H. Jansson, and R. Bergman, Phys. Rev. Lett. 96, 247802 (2006).

[22] S. Pawlus, S. Khodadadi, and A. P. Sokolov, Phys. Rev. Lett. 100, 108103 (2008).

[23] M. Vogel, Phys. Rev. Lett. 101, 225701 (2008).

[24] H. D. Middendorf and S. J. Randall, Phil. Trans. R. Soc. B 290, 639 (1980).

[25] M. C. Bellissent-Funel, J. Teixera, K. F. Bradley, and S.-H. Chen, J. Phys. I (France) 2, 995 (1992).

[26] I. Köper, S. Combet, W. Petry, and M. C. Bellissent-Funel, Eur. Biophys. J. 37, 739 (2008).

[27] A. Gaspar et al., BBA Proteins and Proteomics 1804, 76 (2010).

[28] K. Bradley, S.-H. Chen, M. C. Bellissent-Funel, and H. L. Crespi, Biophys. Chem. 53, 37 (1994).

[29] H. L. Crespi, in Stable Isotopes in The Life Sciences (IAEA, Vienna, 1977), p. 111.

[30] G. Patil, S. Chethana, A. S. Sridevi, and K.S.M. S. Raghavarao, J. Chromatogr. A 1127, 76 (2006).

[31] J. Wuttke et al. (to be published), see http://www.jcns.info/ jens_spheres.

[32] J. Wuttke, FRIDA: fast reliable inelastic data analysis (http://www.messen-und-deuten.de/frida).

[33] J. Wuttke, libkww (http://www.messen-und-deuten.de/ kww); arXiv:0911.4796v1.

[34] S. A. Lusceac, M. R. Vogel, and C. R. Herbers, BBA Proteins and Proteomics 1804, 41 (2010).

[35] S. Khodadadi, A. Malkovskiy, A. Kisliuk, and A.P. Sokolov, BBA Proteins and Proteomics 1804, 15 (2010).

[36] M. Hiebl and R. Maksymiw, Biopolymers 31, 161 (1991).

[37] H. E. Stanley and J. Teixera, J. Chem. Phys. 73, 3404 (1980). 